\title{
Постковидный синдром
}

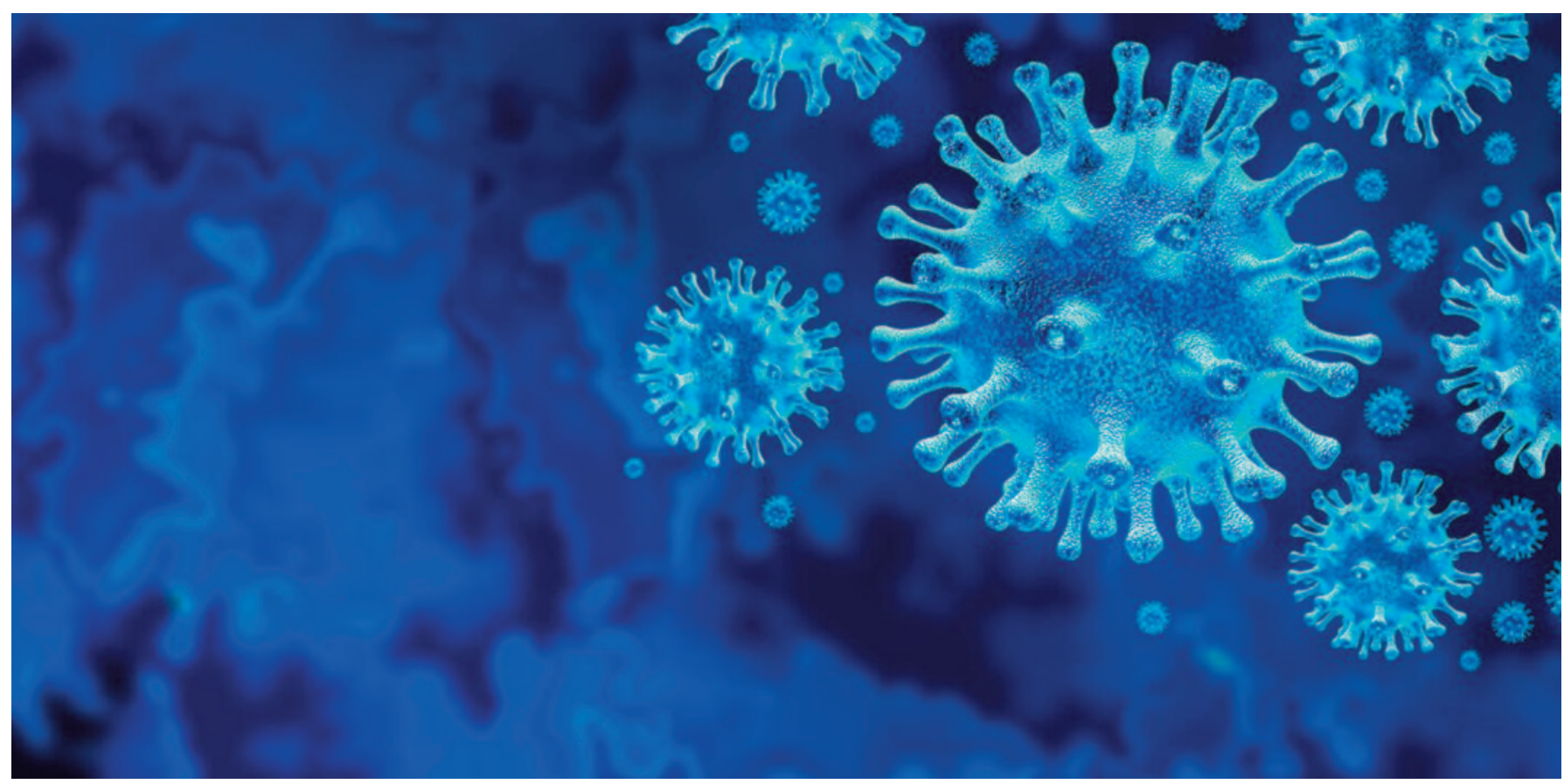

$\mathrm{H}$ овую коронавирусную инфекцию (COVID-19) с уверенностью можно назвать мультисистемным заболеванием с распространенными экстрареспираторными осложнениями, которое проявляется широким спектром клинических симптомов. По тяжести заболевания всех пациентов условно можно разделить на несколько групп:

1. Пациенты с бессимптомным течением заболевания

2. Пациенты с симптомами, изолированные в домашних условиях

3. Пациенты с симптомами, требующими госпитализацию

4. Пациенты с симптомами, требующими госпитализацию в отделения интенсивной терапии и/или проведения искусственной вентиляции легких

Долгосрочные последствия COVID-19 неизвестны. Однако уроки, извлеченные из предыдущих эпидемий, возбудителями которых являлись представители семейства Coronaviridae, демонстрируют нарушения легочной функции, снижение толерантности к физическим нагрузкам, посттравматическое стрессовое расстройство, депрессию и тревогу, что, в целом, приводит к снижению качества жизни. [1] Накапливающиеся данные наблюдений позволяют предположить, что у пациентов, перенесших COVID-19, могут развиться психологические, физические и когнитивные нарушения. Центрами по контролю и профилактике заболеваний США (CDC) предложена следующая классификация: [2]
Таким образом, вводится новый термин: «постковидные состояния» или «пост-острые последствия SARS-CoV-2».

Процесс восстановления после COVID-19 непрерывен. На ранних этапах развития COVID-19 лечение сосредоточено на терапии острых осложнений, ассоциированных с COVID-19, в то время как после острой фазы COVID-19 некоторым пациентам требуется лечение возникших или стойких симптомов. Общепринятых клинических диагностических критериев «длительного COVID» не существует. Однако в Международной классификации болезней 10-го пересмотра добавлен отдельный код: U09.9 - Состояние после COVID-19 неуточненное.

Этот необязательный код позволяет установить связь с COVID-19. [3]

Симптомы постковидных состояний могут влиять на функциональные возможности пациентов. Например, в проспективном исследовании в среднем в течение шести месяцев наблюдали за 177 пациентами (16 стационарных и 161 амбулаторных пациентов) с лабораторно подтвержденной инфекцией SARSCoV-2. У 19\% амбулаторных пациентов были отмечены от одного до двух стойких симптомов через шесть месяцев, у 14\% $\geq 3$ стойких симптомов, а 29\% сообщили о снижении качества жизни. Наиболее частыми стойкими симптомами были утомляемость, потеря обоняния и вкуса, а также одышка. [4] В швейцарском исследовании, объединившем 410 амбулаторных пациентов с легкой формой заболевания, 39\% пациентов сообщили о стойких симптомах через семь-девять месяцев после острой фазы COVID-19. Наиболее частыми симптомами были утомляе-

\begin{tabular}{|l|l|}
\hline Острая фаза COVID-19 & Симптомы COVID-19 в течение 4-х недель от начала заболевания \\
\hline Пост-COVID-состояния & $\begin{array}{l}\text { Широкий спектр симптомов (физических и психических), которые развиваются во время } \\
\text { или после COVID-19 и продолжаются } \geq 2 \text { мес (т. е. } 3 \text { месяца с момента возникновения) } \\
\text { и не объясняются другим альтернативным диагнозом }\end{array}$ \\
\hline Примечание: стадии отражают процесс симптоматического выздоровления и не связаны с активностью вирусной инфекции \\
\hline
\end{tabular}


мость (21\%), потеря вкуса или запаха (17\%), одышка (12\%) и головная боль (10\%). [5] В другом исследовании почти 40\% пациентов не могли вернуться к нормальной повседневной активности через 60 дней после выписки из больницы [6]. Итальянское исследование, в котором наблюдали за 143 пациентами с COVID-19 через 7 недель после выписки из стационара, обнаружило, что 53\% пациентов сообщают об усталости, 43\% - об одышке и $27 \%$ о боли в суставах. [7] По данным британского исследования в течение шести недель после выписки клинически значимое снижение качества жизни согласно опроснику EQ5D наблюдалось у $68,8 \%$ пациентов в группе интенсивной терапии и у $45,6 \%$ пациентов в больничных палатах без интенсивной терапии. [8]

Поскольку пациенты имеют разную степень нарушений после перенесенного заболевания, необходимо разрабатывать индивидуальные планы реабилитации постковидного синдрома в соответствии с возрастом, полом, образом жизни, увлечениями, профессией и физическим состоянием пациентов.

\section{Литература}

1. Ahmed H, Patel K, Greenwood DC, et al. Long-term clinical outcomes in survivors of severe acute respiratory syndrome (SARS) and Middle East respiratory syndrome coronavirus (MERS) outbreaks after hospitalisation or ICU admission: a systematic review and meta-analysis. J Rehabil Med. 2020; 52:00063. https://doi.org/10.2340/16501977-2694

2. World Health Organization. A clinical case definition of post-COVID-19 condition by a Delphi consensus, 6 0ctober 2021. https://www.who.int/publications/i/item/WH0-2019-nCoV-Post_COVID-19_condition-Clinical_case_definition-2021.1 (Accessed on 0ctober 11, 2021).

3. Источник: https://mkb-10.com/ МКБ 10 - Международная классификация болезней 10-го пересмотра (МКБ-10). Дата 0бращения: 25.10.2021

4. Logue JK, Franko NM, McCulloch DJ, et al. Sequelae in Adults at 6 Months After COVID-19 Infection [published correction appears in JAMA Netw 0pen. 2021 Mar 1;4(3):e214572]. JAMA Netw 0pen. 2021;4(2):e210830. Published 2021 Feb 1. doi:10.1001/jamanetworkopen.2021.0830

5. Nehme M, Braillard 0, Chappuis F, Courvoisier DS, Guessous I; CoviCare Study Team. Prevalence of Symptoms More Than Seven Months After Diagnosis of Symptomatic COVID-19 in an Outpatient Setting. Ann Intern Med. 2021;174(9):1252-1260. doi:10.7326/M21-0878

6. Chopra V, Flanders SA, O'Malley M, Malani AN, Prescott HC. Sixty-Day Outcomes Among Patients Hospitalized With COVID-19. Ann Intern Med. 2021;174(4):576-578. doi:10.7326/M20-5661

7. Carfi A, Bernabei R, Landi F; Gemelli Against COVID-19 Post-Acute Care Study Group. Persistent Symptoms in Patients After Acute COVID-19. JAMA. 2020;324(6):603-605. doi:10.1001/jama.2020.12603

8. Halpin SJ, Mclvor C, Whyatt G, et al. Postdischarge symptoms and rehabilitation needs in survivors of COVID-19 infection: A cross-sectional evaluation. J Med Virol. 2021;93(2):1013-1022. doi:10.1002/jmv.26368

Материал принадлежит ООО «ММА«МедиаМдика», любое копирование и использование в коммерческих целях запрещено.

Предназначено исключительно для специалистов здравоохранения. 